(2) Open Access Full Text Article

\title{
A triazole derivative elicits autophagic clearance of polyglutamine aggregation in neuronal cells
}

This article was published in the following Dove Press journal:

Drug Design, Development and Therapy

14 September 2016

Number of times this article has been viewed

\author{
Chang Heng Hsieh' \\ Li-Ching Lee' \\ Wai-Yin Leong' \\ Tsai-Chen Yang' \\ Ching-Fa Yao ${ }^{2}$ \\ Kang Fang' \\ 'Department of Life Science, \\ ${ }^{2}$ Department of Chemistry, \\ National Taiwan Normal University, \\ Taipei, Taiwan
}

\begin{abstract}
Trinucleotide CAG repeat expansion in the coding region of genes has a propensity to form polyglutamine (polyQ) aggregates that contribute to neuronal disorders. Strategies in elevating autophagy to disintegrate the insoluble aggregates without injuring cells have become a major goal for therapy. In this work, a triazole derivative, OC-13, was found accelerating autophagic clearance of polyQ aggregation in human neuroblastoma cells following induction of the enhanced green fluorescence-conjugated chimeric protein that enclosed 79 polyQ repeats (Q79-EGFP). OC-13 accelerated autophagy development and removed nuclear Q79-EGFP aggregates. The increase of Beclin-1, turnover of LC3-I to LC3-II and degradation of p62 supported autophagy activation. Pretreatment of autophagy inhibitor, bafilomycin A1, not only suppressed autophagolysome fusion, but also impeded aggregate eradication. The study also showed that c-Jun N-terminal kinase/Beclin-1 pathway was activated during OC-13 treatment and c-Jun N-terminal kinase inhibitor impaired autophagy and final breakdown. Autophagic clearance of the insoluble aggregates demonstrated the feasibility of OC-13 in alleviating neuronal disorders because of expanded glutamine stretches.
\end{abstract}

Keywords: autophagic flux, polyglutamine, aggregates clearance, triazole, JNK pathway, neuronal disorders, green fluorescence protein

\section{Introduction}

Aggregation of expanded polyglutamine (polyQ) has the propensity to form intraneuronal inclusions. Having strong tendency to aggregate, proteins containing long polyQ stretches produce nuclear inclusions in various regions of the brain and are toxic. ${ }^{1}$ Abnormal expansions of glutamine repeats of ataxin protein and the acquired gain-of-function toxicity contribute to neuronal dysfunction or cell death. $^{2-4}$ The aberrant glutamine repeat expansion during development is dominantly inherited. The acquired aggregates in brain lead to pathogenesis as manifested in a number of neurodegenerative diseases, for example, Huntington's disease and spinocerebellar ataxias. ${ }^{5}$ Due to the insufficient endogenous clearance functions, new strategies aiming to dissolve the abnormal accumulation and obviate neuronal toxicities are constantly needed. Macroautophagy (hereafter referred to as autophagy) is the major catabolic mechanism to clear the accumulated protein aggregates. The coordinated autophagy and proteasome are proposed to ease deleterious effects. Since aggregate-prone polyQ protein is sometimes a poor proteasome substrate, autophagy naturally becomes an ideal alternative to clear the massive aggregation. ${ }^{6}$ Dysfunction of autophagy accumulates abnormal protein and aggravates the pathology of various neuronal disorders. ${ }^{7}$ The induction of autophagy against toxic insults of the mutant aggregate proteins effectively prevents neuronal disorders in animal and cell models. ${ }^{8}$
Correspondence: Kang Fang Department of Life Science, National Taiwan Normal University, 88 Ting Chou Rd, Sec 4, Taipei II6, Taiwan

Tel +886277346293

Fax +8862293I 2904

Email kangfang@ntnu.edu.tw
Drug Design, Development and Therapy 2016:10 2947-2957

(c) (1) (8) ๑ 2016 Hsieh et al. This work is published and licensed by Dove Medical Press Limited. The full terms of this license are avalable at https:/www.dovepress.com/terms.php cc) hereby accept the Terms. Non-commercial uses of the work are permitted without any further permission from Dove Medical Press Limited, provided the work is properly attributed. For permision for commercial use of this work, please see paragraphs 4.2 and 5 of our Terms (https://www.doveppess. com/terms.php). 
Numerous studies showed that autophagic enhancement is a practical approach to abolish the aberrant neuronal aggregation..$^{9,10}$ Recent development to alleviate progressive neurodegeneration of intracellular aggregate-prone proteins by enhancing autophagy has attracted good attention. New drugs to reduce expanded polyQ through autophagy in neuron cells without affecting viabilities are always in pursuit. To further discover more potential drugs to ameliorate aggregates, the established clones of human neuroblastoma cells SK-N-SH were induced to express the N-terminal fragment of TATA-binding domain protein (TBP) enclosing various polyQ repeats tagged with enhanced green fluorescence protein (EGFP) at $3^{\prime}$-end. The triazole derivatives were reported effective in slowing the progression of Parkinson disease. ${ }^{11}$ By screening a panel of compounds with similar structures, the work described identification and evaluation of a new synthetic triazole molecule, OC-13, that dissolves polyQ aggregates while maintaining cell viabilities. The induced autophagy mediated dose-dependent elimination of the aggregated protein containing expanded glutamine stretches. The autophagy inhibitors suppressed polyQ aggregation eradication and blocked drug activity. The autophagic clearance involved activation of c-Jun N-terminal protein kinase 1 and $2(\mathrm{JNK} 1 / 2)$ pathway. The findings provided another candidate chemical with potential to erase the accumulated polyQ aggregates and protect neuronal cells.

\section{Materials and methods Constructs of cDNA with EGFP- conjugated $\triangle \mathrm{C}$-TBP containing 36 and 79 glutamine repeats}

The hemagglutinin-tagged full-length TBP/Q36 and Q79 cDNA inserts in the pGEM-T Easy (Promega Corporation, Fitchburg, WI, USA) and pcDNA5/FRT/TO (Thermo Fisher Scientific, Waltham, MA, USA) vectors were generated similar to previously described. ${ }^{12}$ To manufacture C-terminus-deleted $\triangle C$-TBP/Q79-EGFP construct, the EcoRI (in the multiple cloning sites (MCS) of pGEM-T Easy)-RsaI fragment containing N-terminus $\Delta \mathrm{C}$-TBP/Q79 (203 amino acids) was cut from the cloned TBP/Q79 cDNA and fused in-frame with the EGFP gene between the EcoRI and RsaI sites in the MCS of the pEGFP-N1 vector (Clontech, Mountain View, CA, USA). The Kozak sequence of the EGFP gene was removed by polymerase chain reaction using the site-directed primer CGGGCCCGGGATCCAC
CGGTCGCC $\Delta$ GTGAGCAAGGGCGAGGAGCTG $(\Delta=$ ACCATG). The deleted Kozak sequence was confirmed by DNA sequencing. ${ }^{13}$

\section{Cell lines}

Human neuroblastoma SK-N-SH cells were obtained from the American Type Culture Collection (ATCC, Manassas, VA, USA) and cultured in Dulbecco's Modified Eagle's Medium containing 10\% fetal bovine serum (Thermo Fisher Scientific, Waltham, MA, USA) under humidified atmosphere supplemented with $5 \% \mathrm{CO}_{2}$ at $37^{\circ} \mathrm{C}$. SK-N-SH cells were transfected with $\Delta C-T B P / Q 36$ or $\Delta C-T B P / Q 79$ construct by Lipofectamine 2000 (Thermo Fisher Scientific) and selected by $20 \mu \mathrm{g} / \mathrm{mL}$ of blasticidin (Sigma-Aldrich Co., St Louis, MO, USA) and the stable cell lines established. Expression EGFP-conjugated protein was induced by doxycycline $(20 \mu \mathrm{g} / \mathrm{mL}$, Thermo Fisher Scientific) for 4 days.

\section{Chemicals and materials}

Compound OC-13 was synthesized by adding sodium azide (2.2 $\mathrm{mmol}), 1,3$-bis(ethynyloxy)benzene (1.1 $\mathrm{mmol}$ ), and $\mathrm{CuSO}_{4} \cdot 5 \mathrm{H}_{2} \mathrm{O}(10 \mathrm{~mol} \%)$ to the solution of the 5-(iodomethyl)-3-naphthyloxazolidin-2-one (2.0 mmol) in dimethyl sulfoxide (DMSO; $2 \mathrm{~mL}$ ). The mixture was stirred at $80^{\circ} \mathrm{C}$ until the starting material was consumed as indicated by thin layer chromatography ( 5 hours). After cooling the reaction mixture, crushed ice was added and the resulting precipitate filtered, washed with excess of water and dried to obtain the desired triazole. The crude product was further purified by recrystalizing in methanol. When no precipitate was observed, the triazole was isolated after extraction with ethyl acetate. Further purification was carried out by column chromatography using ethyl acetate/hexane as eluent and white solid obtained, melting point: $193^{\circ} \mathrm{C}-195^{\circ} \mathrm{C} ;{ }^{1} \mathrm{H} \mathrm{NMR}\left(400 \mathrm{MHz}, \mathrm{CDCl}_{3}\right): \delta 7.90$ (s, 2H), 7.84-7.80 (m, 4H), $7.49(\mathrm{~s}, 6 \mathrm{H}), 7.43(\mathrm{t}, J=7.8 \mathrm{~Hz}$, 2H), 7.22-7.41 (m, 3H), $6.16(\mathrm{t}, J=9.8 \mathrm{~Hz}, 3 \mathrm{H}), 5.20-5.14$ $(\mathrm{m}, 6 \mathrm{H}), 4.76(\mathrm{~d}, J=3.5 \mathrm{~Hz}, 4 \mathrm{H}), 4.15(\mathrm{t}, J=9.1 \mathrm{~Hz}$, 2H), 3.93-3.91 (m, 2H); $\left.{ }^{13} \mathrm{C} \mathrm{NMR} \mathrm{(100} \mathrm{MHz,} \mathrm{CDCl}_{3}\right)$ : $159.4,155.8,144.5,134.4,132.9,130.2,129.6,129.0$, $128.6,127.2,126.6,125.6,124.9,124.8,122.0,107.8$, $102.2,71.4,61.6,52.2,50.5$. Mass spectrometry (electrospray ionization) $(\mathrm{m} / \mathrm{z})$ (relative intensity) $745\left[(\mathrm{M}+\mathrm{Na})^{+}\right.$, 100], 736 (10), 459 (3). High resolution mass spectrometry (electrospray ionization) calculated for $\mathrm{C}_{40} \mathrm{H}_{34} \mathrm{~N}_{8} \mathrm{O}_{6} \mathrm{M}^{+}$ 722.2601, found: $745.2504\left(\mathrm{M}^{+}+\mathrm{Na}\right)$. The chemicals as 
tested for clearance were purified to $>98 \%$ and prepared at $10 \mathrm{mM}$ in DMSO for storage at $-20^{\circ} \mathrm{C}$.

The autophagy inhibitors, bafilomycin A1 (Baf A1) and 3-methyladenine (3-MA), and the nucleus staining agent 4',6-diamidino-2-phenylindole (DAPI) were acquired from Sigma-Aldrich. JNK inhibitor Sp600125 was from Selleckchem (Houston, TX, USA). The tet-On system inducer, doxycycline, was from Clontech.

\section{Autophagy detection by LysoTracker staining}

The acidic intracellular compartments of the developing autophagosome were visualized by LysoTracker staining. After incubating with different concentrations of drugs, cells were washed with phosphate-buffered saline (PBS) and added with $100 \mathrm{nM}$ intracellular acidic vesicular dye LysoTracker (Thermo Fisher Scientific) for 15 minutes at $37^{\circ} \mathrm{C}$. The stained cells were washed and observed under the inverted fluorescence microscope. The intracellular acidification was assessed by FACSCalibur (BD Biosciences, Taipei, Taiwan) flow cytometer. The measured red and green fluorescence distributions were converted into ratios (mean FL3/FL1, arbitrarily set to 1 for control samples). The intensities of the collected cells were analyzed by Cell Quest software (BD Biosciences).

\section{Immunoblotting}

The cells were washed with PBS and lysed with radioimmunoprecipitation assay buffer $(150 \mathrm{mM} \mathrm{NaCl}, 50 \mathrm{mM}$ Tris- $\mathrm{HCl}$, $\mathrm{pH}$ 8.0, $25 \mathrm{mM}$ ethylenediaminetetraacetic acid) in $60 \mathrm{~mm}$ petri dishes. The collected cell lysates were stocked under refrigeration of $-20^{\circ} \mathrm{C}$. Equal amounts of protein samples were analyzed by sodium dodecyl sulfate polyacrylamide gel electrophoresis $(8 \%-12 \%)$ and transferred to a nitrocellulose membrane. The blots were incubated with primary antibodies and then probed with peroxidase-conjugated antimouse or -rabbit secondary antibodies at $4^{\circ} \mathrm{C}$ for 18 hours. The antibodies used were against EGFP (Abomics, Turku, Finland), p62/SQSTM1 (Abcam, Cambridge, UK), glyceraldehyde 3-phosphate dehydrogenase, Akt, phospho-Akt ${ }^{473}$ (Genetex, Irvine, CA, USA), S6K, phospho-S6K (Cell Signaling, Danvers, MA, USA), phospho-JNK, JNK, Beclin-1, Bcl-2 (Santa Cruz Biotechnology Inc, Dallas, TX, USA), or LC3 (MBL, Woburn, MA, USA), respectively. The immunoblots were then stained with enhanced chemiluminescence and detected by LAS3000 detector. The blot densitometries were determined using MultiGauge software (Fuji Film, Tokyo, Japan).

\section{Determination of aggregation clearance by fluorescence microscopy}

Cells were stained with DAPI $(10 \mu \mathrm{g} / \mathrm{mL})$ following drug treatment and washed with PBS. The aggregates were measured by Live-Cell Imaging fluorescence microscopy (Leica, Singapore) and quantitated. The emergent spots in 500 EGFP positive cells were counted, converted into percentage ratios and analyzed statistically.

\section{Confocal microscopy}

Cells were seeded in chamber slides and treated with the various concentration of the compound. The slides with attached cells were washed with PBS twice and fixed with 4\% paraformaldehyde in PBS for 15 minutes at room temperature. The slides were then incubated with 1:500 dilution of primary antibody against LC3 at $4^{\circ} \mathrm{C}$ overnight, followed by tetramethylrhodamine-conjugated antimouse or -rabbit secondary antibody of dilution 1:500 overnight. The antibodies were diluted in PBS-T (3.2 mM $\mathrm{Na}_{2} \mathrm{HPO}_{4}, 0.5 \mathrm{mM} \mathrm{KH} \mathrm{PO}_{4}, 1.3 \mathrm{mM} \mathrm{KCl}, 135 \mathrm{mM} \mathrm{NaCl}$, $0.05 \%$ Tween-20, pH 7.4). The slides mounted with $90 \%$ glycerol in PBS were detected with Leica TCS5 confocal spectroscope. The images were analyzed by LAS AF Lite (Leica) software.

\section{Statistical analysis}

The statistical significance was analyzed by one-way analysis of variance analysis. The $P$-value of $<0.05$ was considered statistically significant.

\section{Results}

\section{The identification of the triazole} compound that activated autophagy

To identify candidate chemicals that suppressed aggregates as a result of the induced polyQ proteins, human neuroblastoma cells SK-N-SH were transfected with plasmids encoding EGFP-conjugated $\triangle \mathrm{TBP}$ in which the C-terminus DNA binding domain was deleted. The residual N-terminus TBP fragment contained successive $36(\Delta \mathrm{C}-\mathrm{TBP} / \mathrm{Q} 36-$ EGFP) and 79 glutamine repeats ( $\triangle \mathrm{C}$-TBP/Q79-EGFP), respectively (Figure 1A). The established stable clones, Q36 and Q79, were induced with doxycycline to express the EGFP-conjugated chimeric proteins, $\triangle \mathrm{C}$-TBP-Q36EGFP (Q36-EGFP) and $\triangle \mathrm{C}$-TBP-Q79-EGFP (Q79-EGFP), respectively. The clones as induced were treated with a panel of synthetic triazole chemicals. The formation of autophagosome was monitored by flow cytometry analysis 
A

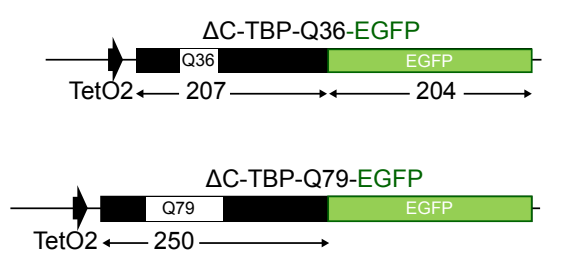

B

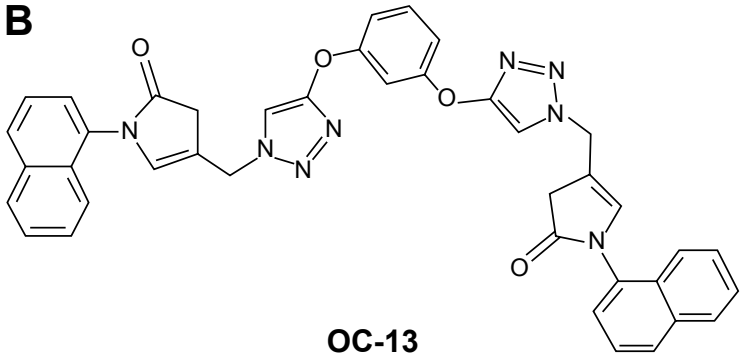

C
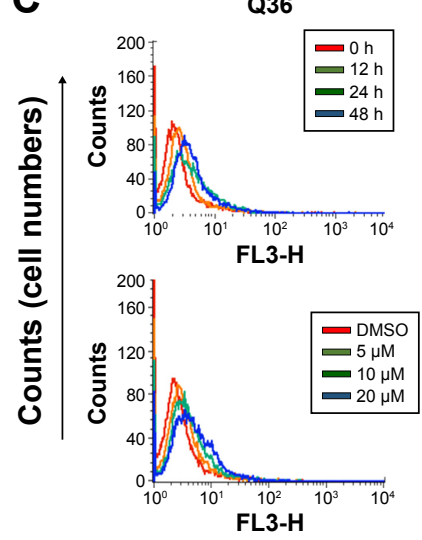

Q79
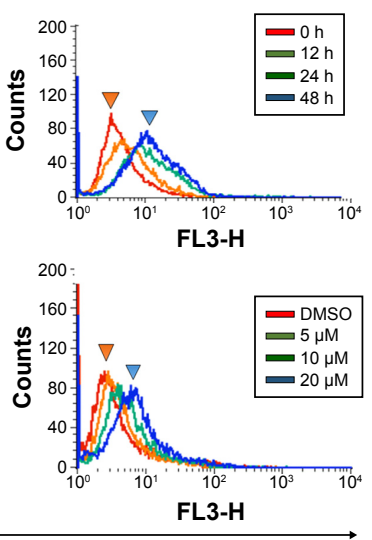

LysoTracker intensity

E

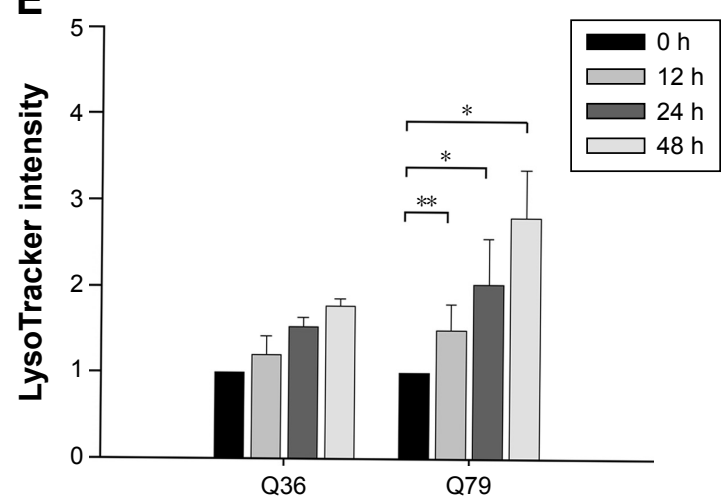

D

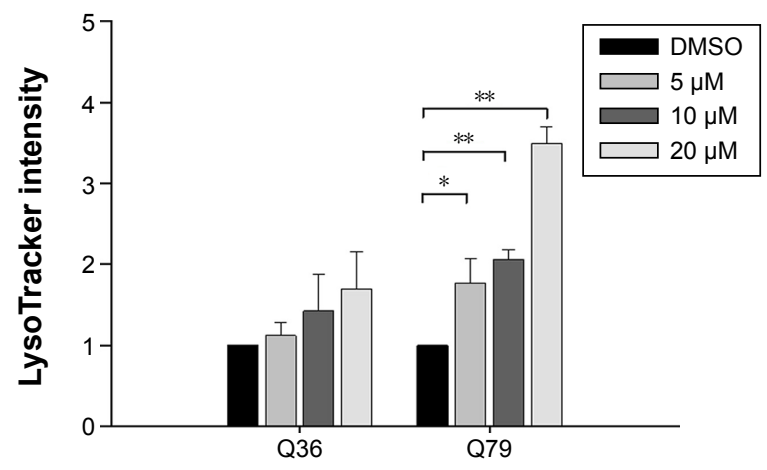

F

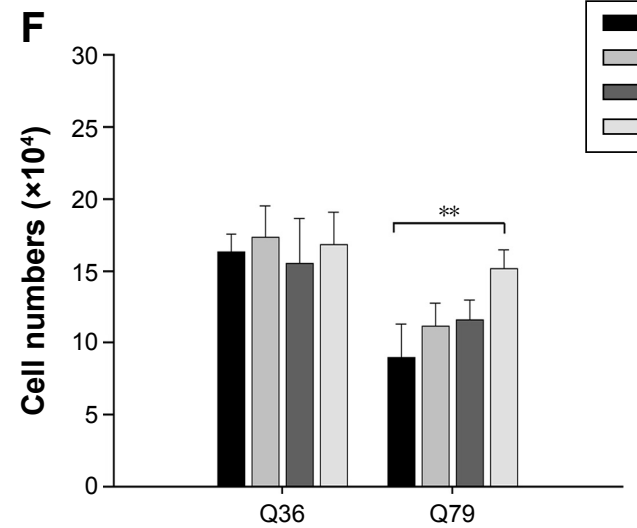

Figure I The identification of the triazole compound that activated autophagy.

Notes: (A) Plasmid construction of $\triangle \mathrm{C}$-TBP/Q36 and Q79. The $\triangle \mathrm{C}$-TBP constructs with enclosure of 36 and 79 glutamine repeats, respectively, by conjugating with EGFP at $3^{\prime}$-end were established. The numbers underneath represent the length of amino acids in each domain. (B) The chemical structure of OC-I3. The synthetic triazole compound, 5,5'-(4,4'-(I,3-phenylene-bis(oxy))-bis(methylene)-bis(I H-I,2,3-triazole-4,I-diyl))-bis(methylene)-bis(3-(naphthalene-I-yl)oxazolidin-2-one), has molecular weight 722.2. (C) Flow cytometric analysis. The top panel shows Lysotracker fluorescence determination by flow cytometric analysis following treatment with $20 \mu M$ of $O C-13$ for $0,12,24$, and $48 \mathrm{~h}$ in the induced Q36 (top left) and Q79 (top right) clones, respectively. The bottom panel indicates flow cytometric analysis of the induced Q36 (bottom left) and Q79 (bottom right) clones after $48 \mathrm{~h}$ treatment with 5, 10, and $20 \mu \mathrm{M}$ and DMSO control. (D) LysoTracker fluorescence enhancement by the increased OC-13 concentrations. Quantitative determination of LysoTracker fluorescence intensities was plotted in Q36 and Q79 clones after $48 \mathrm{~h}$ treatment with 5 , 10 , and $20 \mu \mathrm{M}$. The relative fluorescent levels were obtained by comparing that of DMSO treatment $\left({ }^{*} P<0.05\right.$, ${ }^{* *} P<0.0$ I, Student's $t$-test, compared with vehicle control DMSO). (E) Temporal increases of LysoTracker fluorescence. Quantitative determination of LysoTracker fluorescence intensities in the induced Q36 and Q79 clones following treatment with $20 \mu \mathrm{M} \mathrm{OC}-13$ for 12, 24, and $48 \mathrm{~h}$, respectively. The relative fluorescent levels were obtained by comparing with that at $0 \mathrm{~h}$ treatment $(* P<0.05$, $* * P<0.0 \mathrm{I}$, Student's $t$-test, compared with 0 h). (F) Viable cell number determination. Both Q36 and Q79 clones were cultured onto cell plates ( $2 \times 10^{4}$ cells per well) in Dulbecco's Modified Eagle's Medium supplemented with $10 \%$ fetal bovine serum. The cells were induced with doxycycline for 4 days followed by treating with 5 , 10 , and $20 \mu M$ and DMSO control for $48 \mathrm{~h}$, respectively. Cells were trypsinized and collected. The numbers of viable cells were counted using trypan blue exclusion assay and calculated. Columns stand for mean values of cell numbers of three independent and experiments; bars are standard errors $(* * P<0.0$ I, Student's $t$-test, compared with vehicle control DMSO).

Abbreviations: $\triangle \mathrm{C}$-TBP, C-terminus-truncated TBP; DMSO, dimethyl sulfoxide; EGFP, enhanced green fluorescence protein; h, hours.

in cells stained with LysoTracker. ${ }^{14,15}$ Among them, OC-13 (5,5'-(4,4'-(1,3-phenylene-bis(oxy))-bis(methylene)-bis( $1 H$ 1,2,3-triazole-4,1-diyl))-bis(methylene)-bis(3-(naphthalene1-yl)oxazolidin-2-one); molecular weight 722.2; Figure 1B) enhanced intensities of Lyso Tracker fluorescence (Figure 1C) and the effects were time- and concentration-dependent (Figure 1D and E). The improved autophagy did not affect cell viabilities (Figure $1 \mathrm{~F}$ ). 


\section{OC-I 3 activated autophagic flux and dissolved the insoluble high molecular weight Q79-EGFP aggregates}

Western blot analysis showed that the intensities of high molecular weight Q79-EGFP that accumulated in the stacking gels was reduced after 48 hours treatment. The results indicated dissolution of insoluble aggregates by OC-13 (Figure 2A).
Dose-dependent increase of Beclin-1 and LC3-II/LC3-I ratios along with breakdown of p62/SQSTM1 protein (hereafter referred to as p62) supported autophagic flux during clearance. As lysates of OC-13-treated cells were subjected to a filter trap assay by incubating the blot with EGFP antibody, the collected sodium dodecyl sulfate-insoluble Q79-EGFP aggregates were shown dissipated (Figure 2B) indicating
A

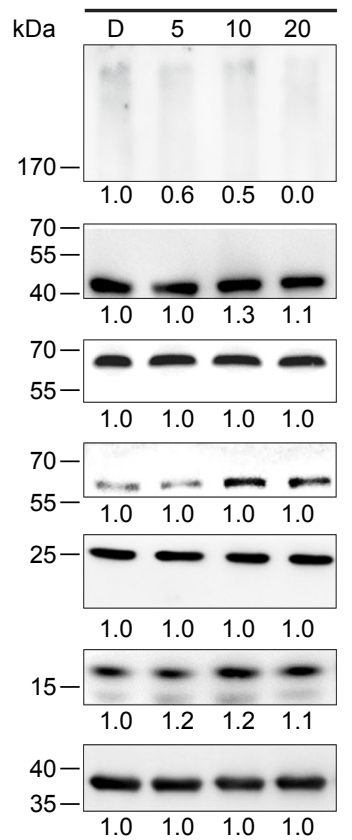

Q79
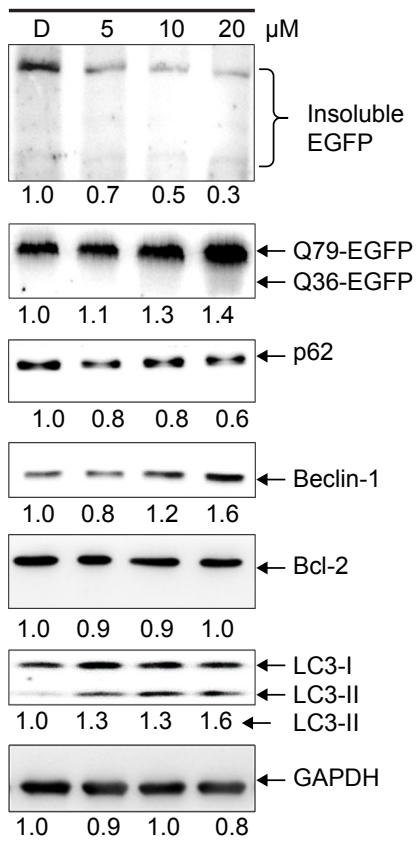

C

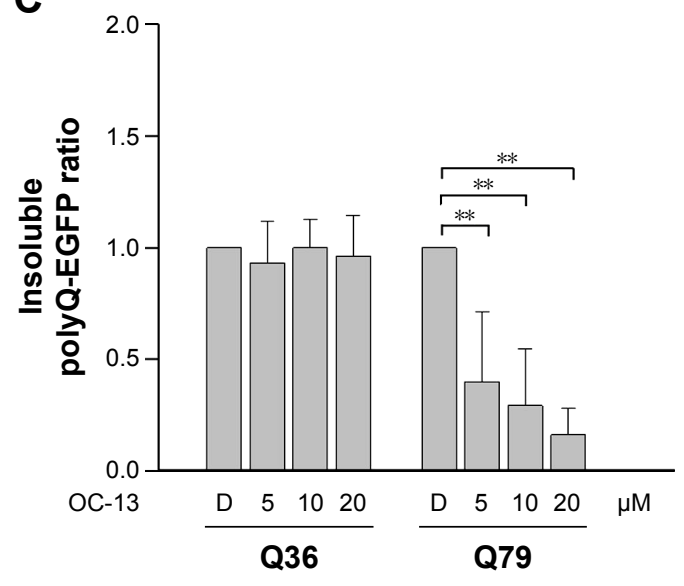

D

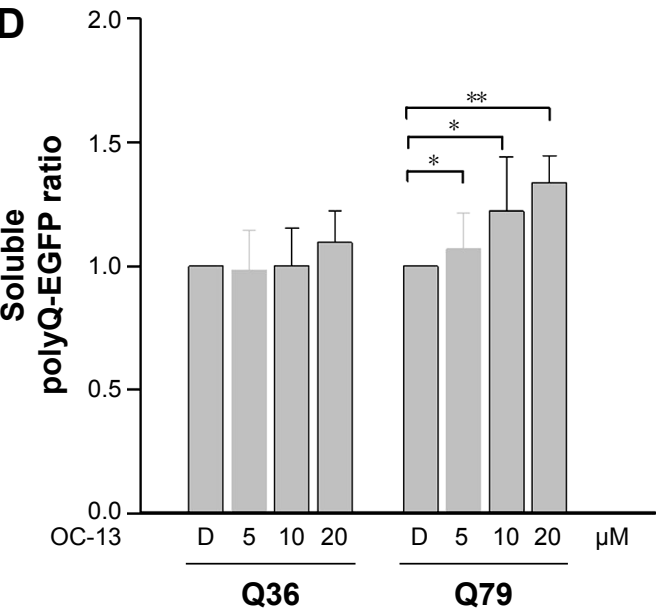

B

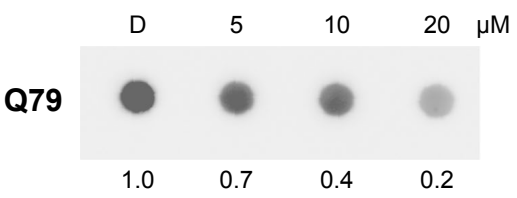

Figure 2 OC-13 activated autophagic flux and dissolved the insoluble Q79-EGFP aggregates.

Notes: (A) Western blot analysis. Protein lysates of the induced clones Q36 and Q79 by treating with 5, 10 , and $20 \mu \mathrm{M}$ of OC-13 for $48 \mathrm{~h}$ were subjected to Western blot analysis. The blots were analyzed for expressions of EGFP, p62, Beclin-I, Bcl-2, and LC3 with GAPDH as loading control. The numbers underneath signify relative intensities compared with that of DMSO vehicle control treatment. The result is a representation of three individual experiments. (B) Filter trap assay. Cells were treated with OC-I3 for $48 \mathrm{~h}$. The insoluble pellets were collected and lysed in sodium dodecyl sulfate buffer. A total of $30 \mu \mathrm{g}$ lysates were filtered through an acetate membrane that was probed with EGFP antibodies to detect the trapped insoluble aggregates. The bottom represents values of densitometric values relative to DMSO control. (C) Densitometric determination of high molecular weight EGFP intensities. The induced Q36 and Q79 clones were treated with 5, I0, and $20 \mu \mathrm{M}$ and DMSO control for $48 \mathrm{~h}$. The densitometric analysis of the insoluble high molecular weight EGFP at each concentration was determined by first normalizing intensities at each concentration to that of the loading control and compared to those treated with DMSO. The error bars stand for standard error from three different experiments ( $* * P<0.0 \mathrm{I}$, unpaired Student's $t$-test as compared with vehicle control DMSO). (D) Densitometric determination of low molecular weight EGFP intensities. The induced Q36 and Q79 clones were treated with 5, 10, and $20 \mu \mathrm{M}$ and DMSO control for $48 \mathrm{~h}$. The densitometric values of soluble low molecular weight EGFP at each concentration were determined as in (C) and converted $(* P<0.01$ and $* * P<0.01$, unpaired Student's $t$-test as compared with vehicle control DMSO).

Abbreviations: D, DMSO; DMSO, dimethyl sulfoxide; EGFP, enhanced green fluorescence protein; GAPDH, glyceraldehyde 3-phosphate dehydrogenase; h, hours. 
enhanced amelioration of the insoluble masses with increasing OC-13 concentrations. Densitometric determination showed that $>50 \%$ of the aggregated Q79-EGFP was dissolved as drug concentration reached $10 \mu \mathrm{M}$. The levels of the soluble EGFP protein in Q79 clone were escalated at the expense of those of the insoluble high molecular weight Q79-EGFP (Figure 2C) and the effects are dose-dependent (Figure 2D). On the other hand, a minor amount of polyQ aggregate was shown in the Q36 clone and OC-13 exerted no variation on the soluble Q36-EGFP protein levels.

\section{Nucleus exclusion and autophagic amelioration of Q79-EGFP aggregates by OC-I 3}

The induced Q79-EGFP aggregate formation in nucleus was proved by confocal microscopy. The emerging EGFP (green) next to nucleus (blue) with increasing OC-13 concentrations indicated that polyQ proteins were excluded from nucleus into cytosol (Figure 3A). The induced autophagy marker puncta LC3 (yellow) that coalesced with the increased cytosolic EGFP indicated that the aggregates were removed from nucleus by compound OC-13 that prompted the autophagosome formation to dissipate the accumulated polyQ (Figure 3B) and the increased drug concentrations accentuated the amelioration (Figure 3C).

\section{The autophagic clearance is related to JNK signaling pathway activation}

To find out the mechanistic aspect of the induced autophagy, Western blot analysis showed that OC-13 not only increased Beclin-1 expression, but also induced phosphorylation of JNK1/2, Akt, and S6K (Figure 4A). Upregulated Beclin-1 activated autophagy. ${ }^{16}$ The increased Akt, S6K, and JNK1/2 phosphorylation in Western blot by incubating with their respective anti-phosphorylated antibodies suggested that the enhanced autophagy is attributed to either activation of
A

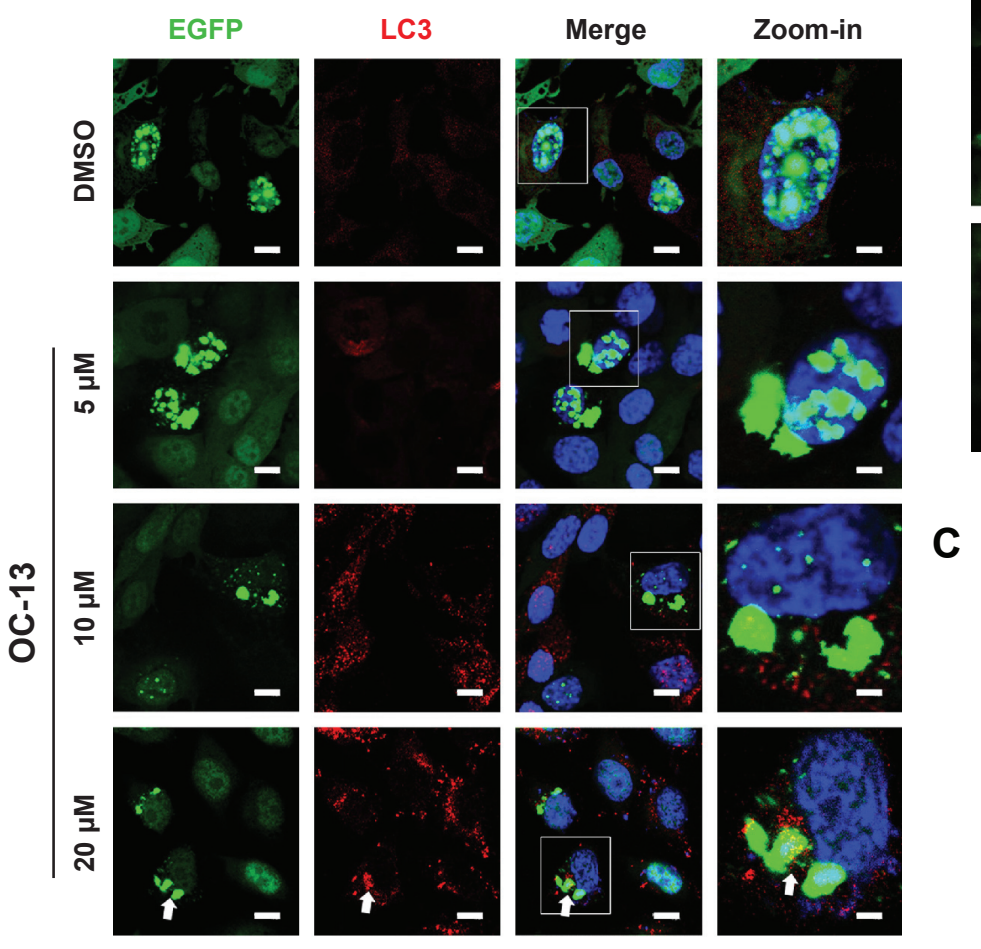

B
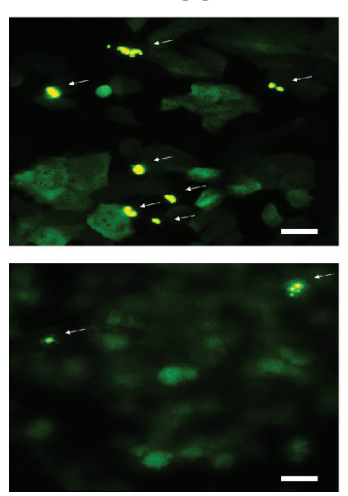

$10 \mu \mathrm{M}$
$5 \mu \mathrm{M}$
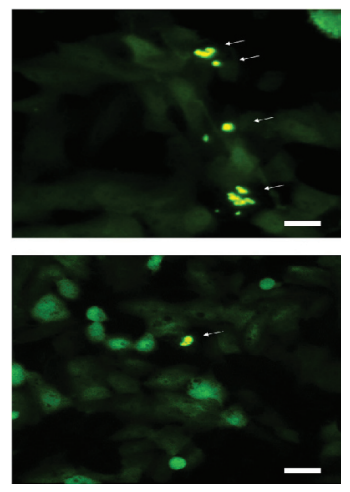

$20 \mu \mathrm{M}$

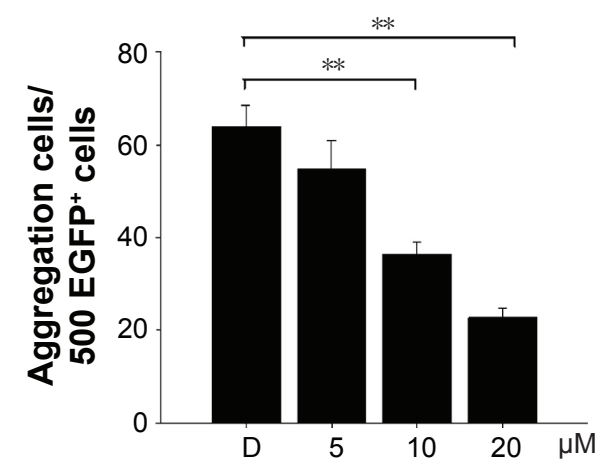

Figure 3 Nucleus exclusion and autophagic amelioration of Q79-EGFP aggregates.

Notes: (A) Confocal microscopy. The induced cells were treated with various concentrations of OC-I3 for 48 h, fixed, incubated with LC3 (red) and EGFP (green) antibodies and counterstained with DAPI (blue). The slides were analyzed by confocal microscopy. The square areas as specified in each image were amplified as zoom-in picture to the right. Symbol arrow represents coalesced EGFP (green) and LC3 (red) antibody-stained markers (scale bar $=10 \mu \mathrm{m}$ ). (B) Fluorescence microscopy. The induced Q79 cells treated with 5,10 , and $20 \mu \mathrm{M}$ of OC-13 as well as DMSO control for $48 \mathrm{~h}$ were analyzed using fluorescence microscopy. Cells with aggregates are indicated as spots (arrow) (scale bar $=20 \mu \mathrm{m}$ ). (C) Quantitative determination of aggregates of various OC-13 concentrations. The aggregates were determined in the induced Q79 cells that were treated with various concentrations of OC-13. The spots numbers were counted in the 500 fluorescent EGFP+ cells at each concentration and plotted $(* * P<0.0$ I, unpaired Student's $t$-test, compared with cells of vehicle control DMSO).

Abbreviations: D, DMSO; DAPI, 4',6-diamidino-2-phenylindole; DMSO, dimethyl sulfoxide; EGFP, enhanced green fluorescence protein; h, hours. 


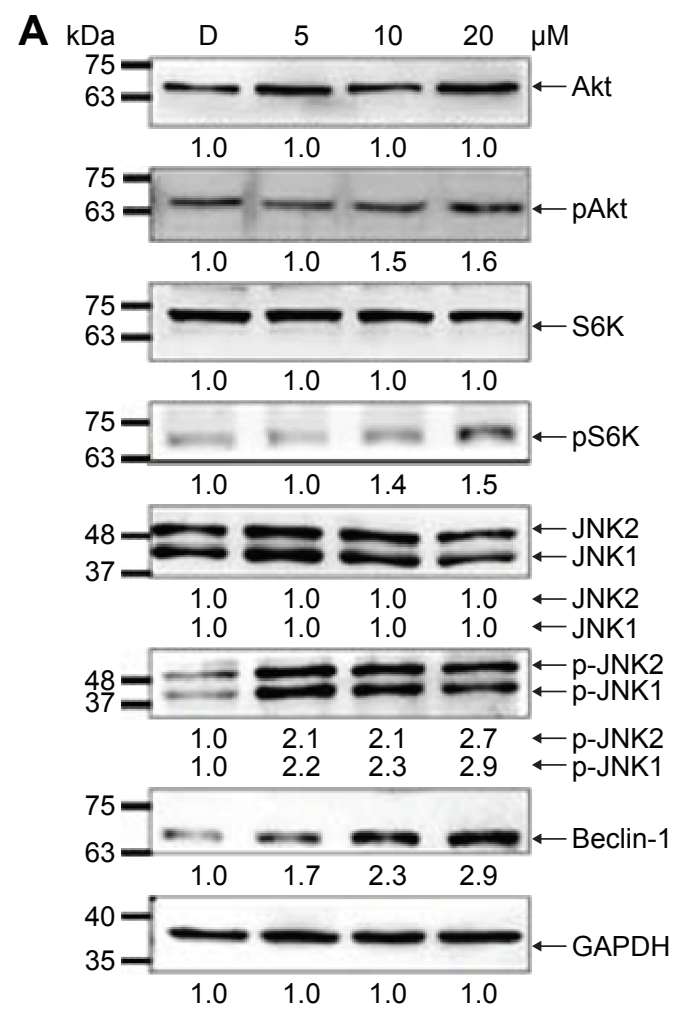

B

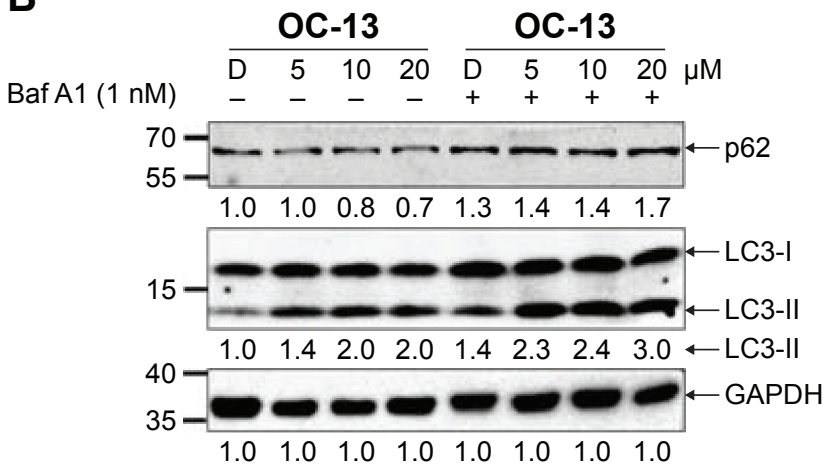

C

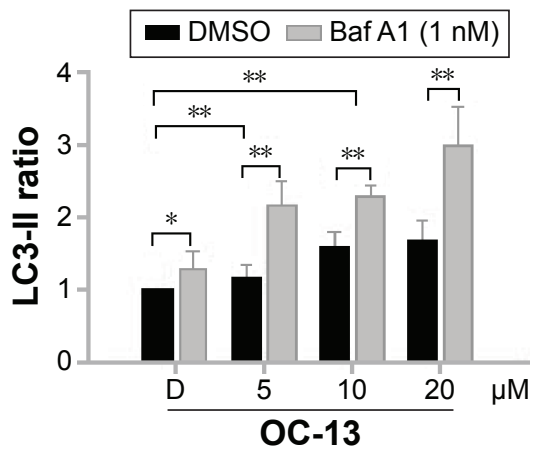

Figure 4 The autophagy pathway is related to JNK signaling activation.

Notes: (A) Western blot analysis. The collected protein lysates of the induced Q79 clone treated with 5, 10, and $20 \mu \mathrm{M}$ of OC-13 plus DMSO control for $48 \mathrm{~h}$ were subjected to Western blot analysis and the membrane incubated with Akt, pAkt, S6K, pS6K, JNKI/2, p-JNKI/2, Beclin-I, and GAPDH antibody, respectively. The bottom numbers represent densitometric determination of ratios of phosphorylated and total proteins relative to DMSO control. The result is a representation of three individual experiments. (B) Incomplete clearance with inhibited autophagy. The induced Q79 clones pretreated with I nM autophagy inhibitor Baf Al (+) or without (-) for I h were incubated with various concentrations of OC-13 for $48 \mathrm{~h}$. The cell lysate proteins as separated by sodium dodecyl sulfate polyacrylamide gel electrophoresis gels were analyzed by immunoblotting with antibodies against p62, LC3, and GAPDH. Densitometry analysis of p62 levels was calculated relative to vehicle DMSO. The relative intensities of LC3-II were converted into ratios and compared with that of DMSO control. (C) Statistical analysis of LC3-II intensity. Densitometry analysis of LC3-II intensity ratio in cells with pretreatment of I nM autophagy inhibitor Baf $\mathrm{Al}(+)$ or without $(-)$ for I h before being incubated with various concentrations of OC-13 for $48 \mathrm{~h}$. The relative intensities of LC3-II were calculated relative to that of vehicle control DMSO alone $(-)$. The statistics were obtained from three individual experiments $(* P<0.05$, $* * P<0.0$ I, Student's $t$-test, compared with vehicle control DMSO).

Abbreviations: Baf AI, bafilomycin AI; D, DMSO; DMSO, dimethyl sulfoxide; GAPDH, glyceraldehyde 3-phosphate dehydrogenase; JNKI/2, c-Jun N-terminal protein kinase I and 2; h, hour.

class III phosphatidyl inositol 3 kinase-Akt axis or JNK1/2 pathway. ${ }^{17,18}$ It is known that phosphorylated Akt-stimulated S6K phosphorylation negatively regulates mammalian target of rapamycin (mTOR)-dependent autophagy pathway. ${ }^{19}$ The results implied that the induced autophagic clearance of Q79-EGFP aggregates involves Beclin-1 and JNK signaling activation and is mTOR independent. ${ }^{20-22}$

Autophagy degrades protein accumulation by sequestering and delivering large aggregates to lysosome. ${ }^{23}$ The increased LC3-II/LC3-I ratio signaled formation of autophagosome prior to fusion to lysosomes. To test if aggregation clearance involves lysosomal activities, the induced Q79 clone was incubated with autophagolysosome inhibitor Baf A1 for 1 hour before treating with OC-13. As an inhibitor of the vacuolar ATPase, Baf A1 accumulates LC3-II, blocks autophagic flux and impairs fusion of autophagosome and lysosome. In addition, the delayed turnover of cargo adaptor p62 meant impaired autophagic degradation of ubiquitinated proteins..$^{24,25}$ Pretreatment Baf A1 increased endogenous LC3-II/LC3-I that was further exacerbated by OC-13. The impaired lysosomal acidification by Baf A1 resulted in failure of LC3-II protein turnover. ${ }^{26,27}$ Elevation in the endogenous and conspicuous retention of LC3-II supported failure of autophagosome to fuse with lysosomes (Figure 4B). Baf A1 incapacitated turnover of $\mathrm{p} 62$ and hampered autolysosomes fusion (Figure 4B) and blocked degradation of LC3-II of Q79 (Figure 4C).

\section{Inhibition autophagy flux blocked clearance of the Q79-EGFP aggregates by $\mathrm{OC}-13$}

To ascertain that OC-13 triggered JNK-dependent autophagy that reduced expanded polyQ protein aggregates, pretreatment of JNK inhibitor Sp600125, and autophagy inhibitors 
A

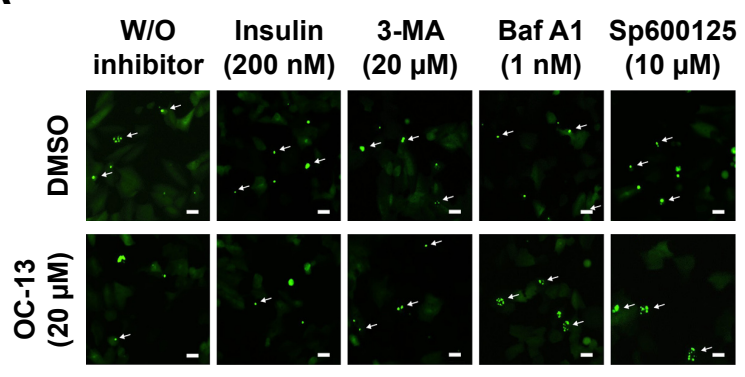

B

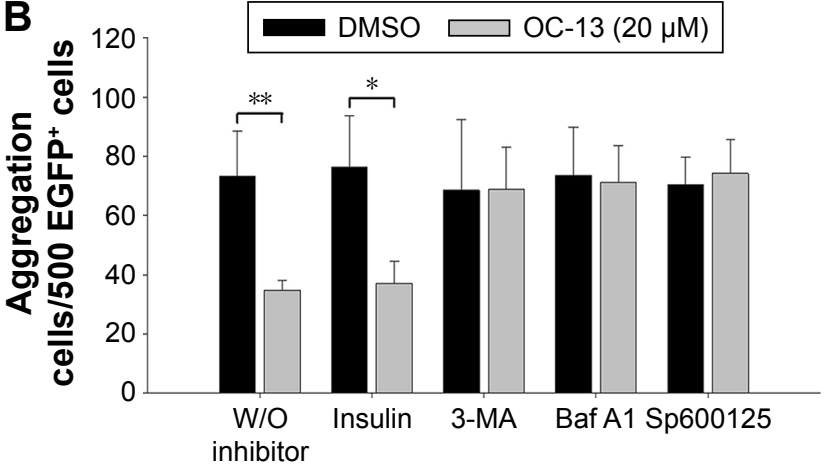

D

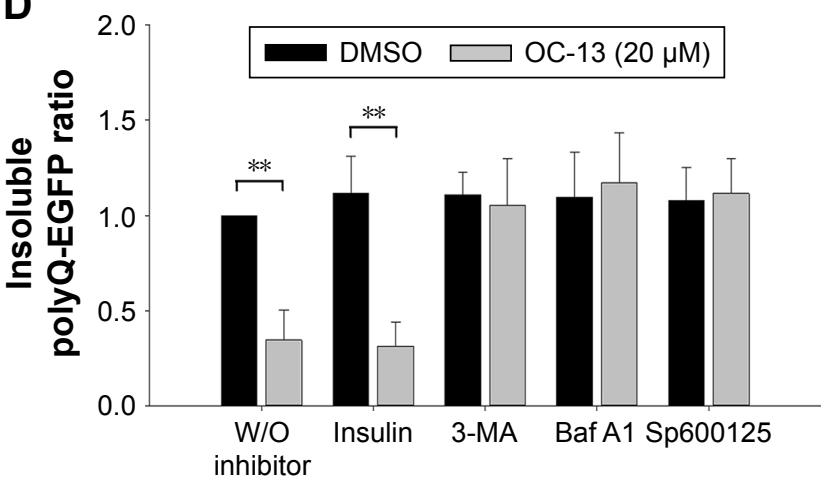

C

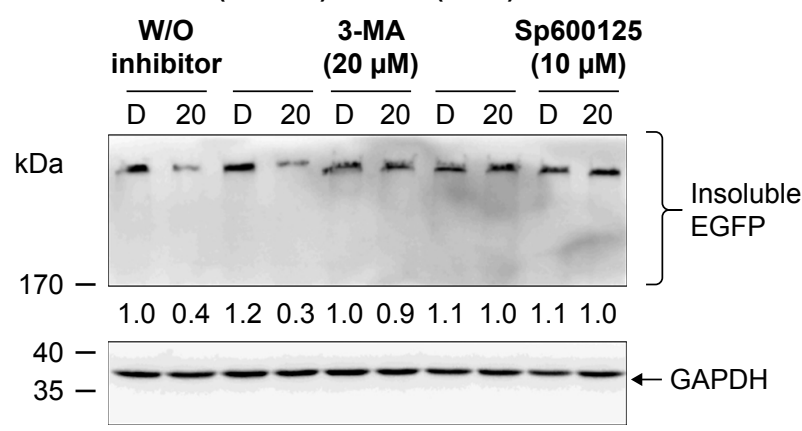

1.01 .01 .01 .01 .01 .01 .01 .01 .01 .0

Figure 5 Inhibition autophagy flux blocked clearance of the Q79-EGFP aggregates.

Notes: (A) Aggregation cell number determination by fluorescence microscopy. The induced Q79 cells were incubated with $200 \mathrm{nM}$ insulin, $20 \mu \mathrm{M} 3-\mathrm{MA}$, I nM Baf AI, and $10 \mu \mathrm{M}$ JNK inhibitor Sp600 I25, respectively, for I h before being treated with vehicle control DMSO (top panel) and $20 \mu \mathrm{M}$ of OC-I3 (bottom panel) for I $2 \mathrm{~h}$. The aggregate spots of fluorescence microscopy were pointed out (arrow) (scale bar $=20 \mu \mathrm{m}$ ). (B) Quantitative determination of aggregate numbers. By counting the aggregate numbers in fluorescent cells, the aggregates in the induced Q79 clone with pretreatment of various inhibitors as specified before being treated with OC-13 (20 $\mu$ M) or DMSO for $12 \mathrm{~h}$. The total aggregates as counted in $500 \mathrm{EGFP}^{+}$cells of OC-I 3 treatment were compared with those of DMSO control (**P<0.0I, $* P<0.05$, Student's $t$-test). (C) Western blot analysis of the insoluble proteins. The induced Q79 cells were incubated with $200 \mathrm{nM}$ insulin, $20 \mu \mathrm{M} 3-\mathrm{MA}$, I nM Baf AI, and I0 $\mu$ M Sp600I25, respectively, for I h before being treated with $20 \mu \mathrm{M}$ of OC-I3 or DMSO for $48 \mathrm{~h}$. The cell lysate proteins as separated by sodium dodecyl sulfate polyacrylamide gel electrophoresis gels were analyzed by immunoblotting for expressions of EGFP with GAPDH as loading control. The numbers underneath signify relative intensities by comparing with that of DMSO treatment. (D) Densitometric determination of high molecular weight EGFP levels. The densitometric values of the insoluble high molecular weight EGFP at each concentration were determined by first normalizing to the loading control and compared with those treated with DMSO without inhibitor pretreatment. The error bars stand for $S E$ from three different experiments ( $* * P<0.0$ I, unpaired Student's $t$-test as compared with vehicle control DMSO).

Abbreviations: Baf AI, bafilomycin AI; D, DMSO; DMSO, dimethyl sulfoxide; EGFP, enhanced green fluorescence protein; GAPDH, glyceraldehyde 3-phosphate dehydrogenase; JNK, c-Jun N-terminal protein kinase; 3-MA, 3-methyladenine; h, hour; W/O, without.

Baf A1 and 3-MA impaired clearance of the green fluorescent aggregation. On the other hand, insulin failed to impede the autophagy flux from clearing the accumulated aggregates (Figure 5A and B) that were supported by Western blot analysis (Figure 5C and D). The results of confocal microscopy elicited that the autophagy inhibitors and Sp600125 impaired elimination of Q79-EGFP aggregates (Figure 6A). More statistical analysis showed that 3-MA and JNK inhibitor diminished LC3 puncta, whereas the accumulated autophagosomes by Baf A1 failed to fuse with lysosome to eradicate polyQ proteins (Figure 6B).

\section{Discussion}

New strategies to eliminate mutant protein aggregates are growing and their comprehensive mechanisms elucidated increasingly. ${ }^{28}$ The misfolded mutant proteins tend to aggregate and become toxic. Because the insoluble aggregated form is at times a poor proteasome substrate, autophagy turns out a viable and major alternative to ease the pathogenesis. Autophagy dysfunction exacerbates the progress of various neuronal degeneration. Activation of autophagic clearance promises to mitigate the disorders. ${ }^{8,29}$

The autophagic pathway cleared mutant polyQ of androgen receptor from motor neuronal cells that caused spinal and bulbar muscular atrophy..$^{30}$ Autophagy upregulation is known to be beneficial to reduce aggregate-prone polyQ proteins that protect cell integrity with implication of alleviating neurodegeneration. ${ }^{31}$ The establishment of neuroblastoma cells with inducible Q79-EGFP serves as a viable tool to assess aggregation development, as compared with that of 

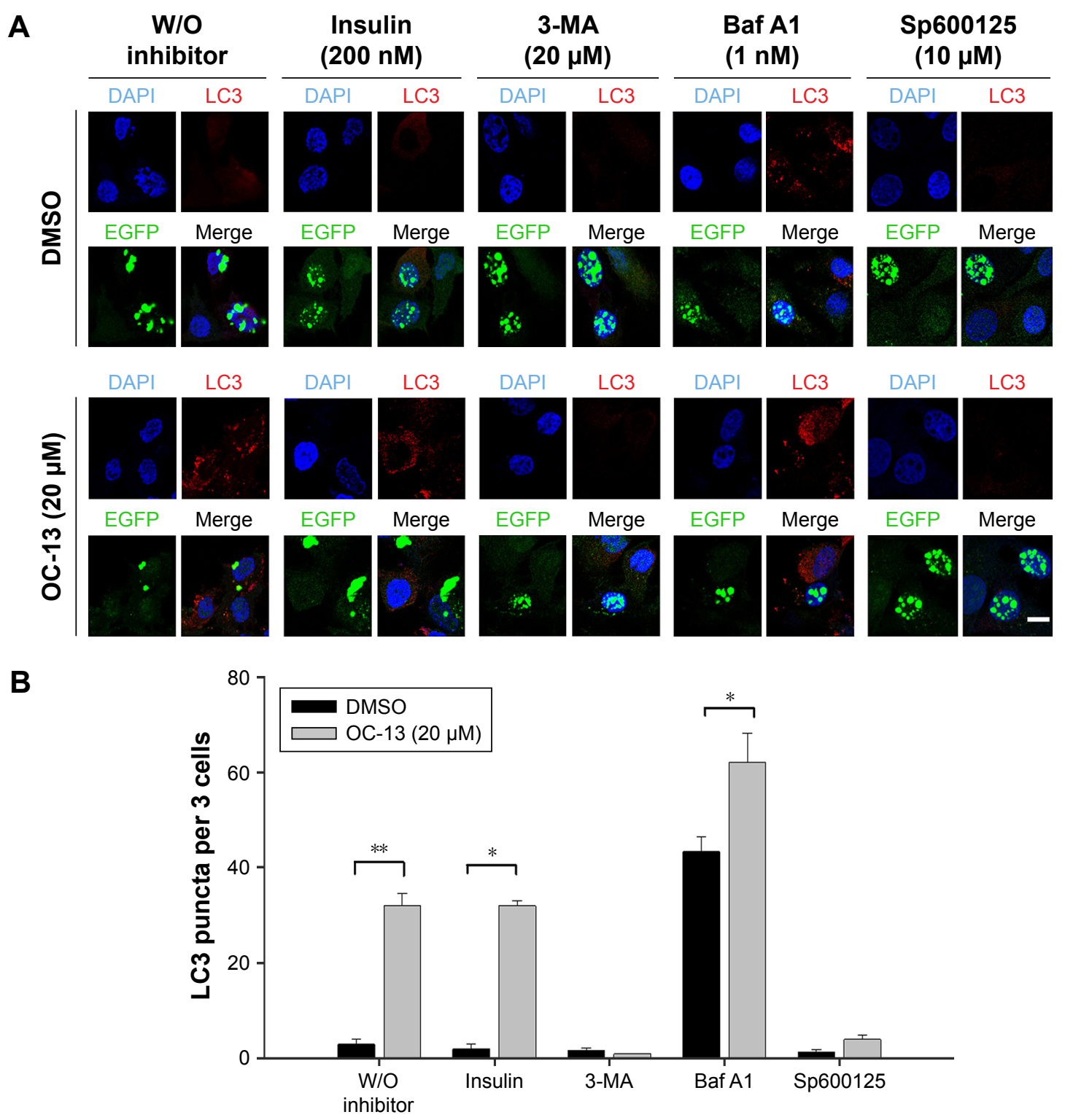

Figure 6 Inhibition lysosome fusion with autophagosome impaired clearance of the Q79-EGFP aggregates.

Notes: (A) Puncta determination by confocal microscopy. The induced Q79 cells were incubated with $200 \mathrm{nM}$ insulin, $20 \mu \mathrm{M} 3-\mathrm{MA}, \mathrm{I} \mathrm{nM}$ Baf AI, and I0 $\mu$ M Sp600I25, respectively, for I h before being treated with DMSO as vehicle control (top panel) or $20 \mu \mathrm{M}$ of OC-I3 (bottom panel) for I $2 \mathrm{~h}$. The cells were fixed, incubated with LC 3 (red) and EGFP (green) antibodies and counterstained with DAPI (blue). The slides were analyzed by confocal microscopy (scale bar $=10 \mu \mathrm{m}$, and applied to all images). (B) Quantitative determination of LC3 puncta per three cells. To determine puncta in three cells, the numbers in the induced Q79 clone with pretreatment of various inhibitors were counted as specified before treatment with OC-13 $(20 \mu \mathrm{M})$ or DMSO for $12 \mathrm{~h}$. The total puncta in three cells by OC-13 treatment were compared with DMSO $(* * P<0.01$, $* P<0.05$, Student's t-test).

Abbreviations: Baf AI, bafilomycin AI; DAPI, 4',6-diamidino-2-phenylindole; DMSO, dimethyl sulfoxide; EGFP, enhanced green fluorescence protein; 3-MA, 3-methyladenine; h, hour; W/O, without.

Q36-EGFP. The results showed that a triazole compound OC-13 removed the induced nuclear polyQ aggregation in Q79 clone as indicated by Western blot analysis and confocal microscopy. Activation of autophagy by OC-13 suppressed polyQ accumulation in cultured cells, in which the targeted cytoplasmic components is enclosed by double-membrane autophagosome that subsequently fused with lysosomes for degrading mutant aggregates. ${ }^{32}$ The compound induced autophagy by activating Beclin-1. Then cargo adaptor p62 binds ubiquitinated proteins and recruits LC3-I. The resultant turnover of LC3-I to LC-II and p62 degradation facilitated assembly of autophagy machinery as autolysosome. The presence of autophagy inhibitor Baf A1 suppressed the final fusion from forming autophagolysosomes and hampering aggregates from being cleared.

Expansion of glutamine tracts exceeding a normal range of elongated polyQ stretch at its amino-terminus of the protein produces a mutant form. The aberrant protein with 
expanded glutamine repeats formed aggregates in neurons. ${ }^{33}$ While the induced nuclear Q79 aggregations remained intact under DMSO control, OC-13 activated JNK that phosphorylated Bcl-2 and dissociated from Beclin-1 and helped autophagy development. ${ }^{28}$ Strategies to ease mutant aggregates confer protection against toxicity in cell and animal models and interrupt the disease pathogenesis. ${ }^{34}$ The approach was applied in a number of pathologies that include cancer and neurodegeneration. ${ }^{28}$ Several pharmacological inducers to upregulate autophagy in mammals are activated by mTOR-dependent or mTOR-independent pathways. ${ }^{35}$ Rapamycin or its analog rapalogs removes expanded repeats of Htt through mTOR pathway. ${ }^{11,36,37}$ Phosphorylated Akt activated mTOR complexes, which in turn downregulated autophagy. ${ }^{22,38,39}$ On the other hand, calpastatin and rilmenidine removed polyQ aggregation such as mutant $\mathrm{Htt}$ and $\alpha$-synuclein by activating JNK. Likewise, OC-13-induced autophagy is independent of mTOR by activating JNK1/ Beclin-1 pathway through phosphorylating JNK1/2. ${ }^{17,37}$ OC-13 induced autophagy through activation of JNK1/2, which in turn reduced the insoluble polyQ aggregation. Treatment with autophagy and JNK inhibitors completely protected aggregates against autophagy-mediated clearance. The results suggested that the nontoxic OC-13 is feasible as a candidate drug for efficacious relief of neuronal disorders and the clearance of the aberrant protein accumulation depends on autophagy activation.

It is yet unclear if clearance of aggregates may be beneficial or not. Nonetheless, as autophagy upregulation becomes a feasible approach to alleviate polyQ-related disease, new strategies to accelerate the clearance of the mutant proteins are needed and growing. ${ }^{40}$ The effective autophagic substrates like mutant proteins as activated by small molecules constitute a promising therapeutic approach. ${ }^{41}$ The work showed that the synthetic triazole acts as autophagy enhancer and can be protective. By targeting misfolded protein for clearance without injuring cells, OC-13 may have good potential to reverse neurodegenerative disorders.

\section{Conclusion}

A triazole OC-13 stimulated autophagy, removed the accumulated nucleus aggregates made of EGFP-conjugated protein with enclosure of 79 polyQ repeats. The attenuated aggregated protein intensities were demonstrated by fluorescence microscopy, confocal microscopy, and Western blot analysis. The induced autophagy clearance involved the activation of JNK, whose inhibitor blocked the amelioration. The results provided a new dimension of the new compound that promises a new approach to clear polyQ aggregation in neuronal cells.

\section{Acknowledgments}

The work is supported by grants from the National Taiwan Normal University (102T3040B2, 103T3040C2, and 104T3040D2). Scholarship to Chang Heng Hsieh by Taiwan Foundation of Rare Disorders is appreciated. Technical assistance of confocal laser microscopy from College of Life Science and Instrumentation Center, National Taiwan University, and fluorescence and confocal laser microscopy from Department of Life Science and Instrumentation Center, National Taiwan Normal University is acknowledged.

\section{Disclosure}

The authors report no conflicts of interest in this work.

\section{References}

1. Wong E, Cuervo AM. Autophagy gone awry in neurodegenerative diseases. Nat Neurosci. 2010;13(7):805-811.

2. Duarte-Silva S, Silva-Fernandes A, Neves-Carvalho A, Soares-Cunha C, Teixeira-Castro A, Maciel P. Combined therapy with m-TOR-dependent and -independent autophagy inducers causes neurotoxicity in a mouse model of Machado-Joseph disease. Neuroscience. 2016;313: 162-173.

3. del Cano-Espinel M, Acebes JR, Sanchez D, Ganfornina MD. Lazarillo-related Lipocalins confer long-term protection against type I spinocerebellar ataxia degeneration contributing to optimize selective autophagy. Mol Neurodegener. 2015;10:11.

4. Duncan C, Papanikolaou T, Ellerby LM. Autophagy: polyQ toxic fragment turnover. Autophagy. 2010;6(2):312-314.

5. Takahashi T, Katada S, Onodera O. Polyglutamine diseases: where does toxicity come from? What is toxicity? Where are we going? J Mol Cell Biol. 2010;2(4):180-191.

6. Mizushima N, Klionsky DJ. Protein turnover via autophagy: implications for metabolism. Annu Rev Nutr. 2007;27:19-40.

7. Martin DD, Ladha S, Ehrnhoefer DE, Hayden MR. Autophagy in Huntington disease and huntingtin in autophagy. Trends Neurosci. 2015; 38(1):26-35.

8. Rubinsztein DC, Gestwicki JE, Murphy LO, Klionsky DJ. Potential therapeutic applications of autophagy. Nat Rev Drug Discov. 2007;6(4): 304-312.

9. Sarkar S, Rubinsztein DC. Huntington's disease: degradation of mutant huntingtin by autophagy. FEBS J. 2008;275(17):4263-4270.

10. Wei PF, Zhang L, Nethi SK, et al. Accelerating the clearance of mutant huntingtin protein aggregates through autophagy induction by europium hydroxide nanorods. Biomaterials. 2014;35(3):899-907.

11. Ray S, Liu M. Current understanding of LRRK2 in Parkinson's disease: biochemical and structural features and inhibitor design. Future Med Chem. 2012;4(13):1701-1713.

12. Lee LC, Chen CM, Chen FL, et al. Altered expression of HSPA5, HSPA8 and PARK7 in spinocerebellar ataxia type 17 identified by 2-dimensional fluorescence difference in gel electrophoresis. Clin Chim Acta. 2009;400(1-2):56-62.

13. Lee L-C, Chen C-M, Wang P-R, Su M-T, Lee-Chen G-J, Chang C-Y. Role of high mobility group box 1 (HMGB1) in sca17 pathogenesis. PLoS One. 2014;9(12):e115809.

14. Chikte S, Panchal N, Warnes G. Use of LysoTracker dyes: a flow cytometric study of autophagy. Cytometry A. 2014;85(2):169-178. 
15. Warnes G. Measurement of autophagy by flow cytometry. Curr Protoc Cytom. 2014;68:9.45.1-9.45.10.

16. Mealer RG, Murray AJ, Shahani N, Subramaniam S, Snyder SH. Rhes, a striatal-selective protein implicated in Huntington disease, binds Beclin-1 and activates autophagy. J Biol Chem. 2014;289(6): 3547-3554.

17. Sarkar S. Regulation of autophagy by mTOR-dependent and mTORindependent pathways: autophagy dysfunction in neurodegenerative diseases and therapeutic application of autophagy enhancers. Biochem Soc Trans. 2013;41(5):1103-1130.

18. Sarkar S. Chemical screening platforms for autophagy drug discovery to identify therapeutic candidates for Huntington's disease and other neurodegenerative disorders. Drug Discov Today Technol. 2013;10(1): e137-e144.

19. Liu Q, Thoreen C, Wang J, Sabatini D, Gray NS. mTOR mediated anticancer drug discovery. Drug Discov Today Ther Strateg. 2009;6(2): 47-55.

20. Floto RA, Sarkar S, Perlstein EO, Kampmann B, Schreiber SL, Rubinsztein DC. Small molecule enhancers of rapamycin-induced TOR inhibition promote autophagy, reduce toxicity in Huntington's disease models and enhance killing of mycobacteria by macrophages. Autophagy. 2007;3(6):620-622.

21. Renna M, Jimenez-Sanchez M, Sarkar S, Rubinsztein DC. Chemical inducers of autophagy that enhance the clearance of mutant proteins in neurodegenerative diseases. $J$ Biol Chem. 2010;285(15): 11061-11067.

22. Roscic A, Baldo B, Crochemore C, Marcellin D, Paganetti P. Induction of autophagy with catalytic mTOR inhibitors reduces huntingtin aggregates in a neuronal cell model. J Neurochem. 2011;119(2):398-407.

23. Ravikumar B, Duden R, Rubinsztein DC. Aggregate-prone proteins with polyglutamine and polyalanine expansions are degraded by autophagy. Hum Mol Genet. 2002;11(9):1107-1117.

24. Rossi M, Munarriz ER, Bartesaghi S, et al. Desmethylclomipramine induces the accumulation of autophagy markers by blocking autophagic flux. J Cell Sci. 2009;122(Pt 18):3330-3339.

25. Zhang D, Zhang W, Li D, Fu M, Chen R, Zhan Q. GADD45A inhibits autophagy by regulating the interaction between BECN1 and PIK3C3. Autophagy. 2015;11(12):2247-2258.

26. Mauvezin C, Neufeld TP. Bafilomycin A1 disrupts autophagic flux by inhibiting both V-ATPase-dependent acidification and Ca-P60A/ SERCA-dependent autophagosome-lysosome fusion. Autophagy. 2015; 11(8):1437-1438.

27. Zhu S, Rea SL, Cheng T, et al. Bafilomycin A1 attenuates osteoclast acidification and formation, accompanied by increased levels of SQSTM1/p62 Protein. J Cell Biochem. 2016;117(6):1464-1470.
28. Jimenez-Sanchez M, Thomson F, Zavodszky E, Rubinsztein DC. Autophagy and polyglutamine diseases. Prog Neurobiol. 2012;97(2): $67-82$.

29. Williams A, Jahreiss L, Sarkar S, et al. Aggregate-prone proteins are cleared from the cytosol by autophagy: therapeutic implications. Curr Top Dev Biol. 2006;76:89-101.

30. Rusmini P, Bolzoni E, Crippa V, et al. Proteasomal and autophagic degradative activities in spinal and bulbar muscular atrophy. Neurobiol Dis. 2010;40(2):361-369.

31. Watson LM, Scholefield J, Greenberg LJ, Wood MJ. Polyglutamine disease: from pathogenesis to therapy. S Afr Med J. 2012;102(6): 481-484.

32. Settembre C, Fraldi A, Jahreiss L, et al. A block of autophagy in lysosomal storage disorders. Hum Mol Genet. 2008;17(1):119-129.

33. Cortes CJ, La Spada AR. Autophagy in polyglutamine disease: Imposing order on disorder or contributing to the chaos? Mol Cell Neurosci. 2015;66(Pt A):53-61.

34. Sarkar S, Krishna G, Imarisio S, Saiki S, O'Kane CJ, Rubinsztein DC. A rational mechanism for combination treatment of Huntington's disease using lithium and rapamycin. Hum Mol Genet. 2008;17(2):170-178.

35. Baldo B, Soylu R, Petersen A. Maintenance of basal levels of autophagy in Huntington's disease mouse models displaying metabolic dysfunction. PLoS One. 2013;8(12): 833050

36. Sarkar S, Ravikumar B, Floto RA, Rubinsztein DC. Rapamycin and mTOR-independent autophagy inducers ameliorate toxicity of polyglutamine-expanded huntingtin and related proteinopathies. Cell Death Differ. 2009;16(1):46-56.

37. Rose C, Menzies FM, Renna M, et al. Rilmenidine attenuates toxicity of polyglutamine expansions in a mouse model of Huntington's disease. Hum Mol Genet. 2010;19(11):2144-2153.

38. Qi M, Zhou H, Fan S, et al. mTOR inactivation by ROS-JNK-p53 pathway plays an essential role in psedolaric acid B induced autophagydependent senescence in murine fibrosarcoma L929 cells. Eur $J$ Pharmacol. 2013;715(1-3):76-88.

39. Song YM, Song SO, Jung YK, et al. Dimethyl sulfoxide reduces hepatocellular lipid accumulation through autophagy induction. Autophagy. 2012;8(7):1085-1097.

40. Sarkar S, Ravikumar B, Rubinsztein DC. Autophagic clearance of aggregate-prone proteins associated with neurodegeneration. Methods Enzymol. 2009;453:83-110.

41. Nedelsky NB, Todd PK, Taylor JP. Autophagy and the ubiquitinproteasome system: collaborators in neuroprotection. Biochim Biophys Acta. 2008;1782(12):691-699.
Drug Design, Development and Therapy

\section{Publish your work in this journal}

Drug Design, Development and Therapy is an international, peerreviewed open-access journal that spans the spectrum of drug design and development through to clinical applications. Clinical outcomes, patient safety, and programs for the development and effective, safe, and sustained use of medicines are the features of the journal, which

\section{Dovepress}

has also been accepted for indexing on PubMed Central. The manuscript management system is completely online and includes a very quick and fair peer-review system, which is all easy to use. Visit http://www.dovepress.com/testimonials.php to read real quotes from published authors. 